\title{
Phenotypic characterization of Trichosporon species from clinical isolates
}

\author{
Premamalini T ${ }^{1}$, Anitha S. ${ }^{2}$, Mohana Priya ${ }^{3}$, Kindo A. J. ${ }^{4}$ \\ ${ }^{1}$ Dr. Premamalini. T, Associate Professor, ${ }^{2}$ Dr. Anitha. S. Clinical Research fellow, ${ }^{3}$ Dr. Mohana Priya K, Lab \\ Technologist, ${ }^{4}$ Dr. Anupma Jyoti Kindo, Professor and Head, all authors are affiliated with Department of Microbiology, \\ Sri Ramachandra Medical College and Research Institute, Chennai, Tamilnadu, India
}

Corresponding author: Dr Premamalini T, Email:drtpremamalini@gmail.com

\begin{abstract}
Introduction: Trichosporon spp. arepresent as normal flora of skin and perigenital area in humans. They can cause invasive trichosporonosis in immuno-compromised individuals. Trichosporon infections are difficult to treat, since they do not respond to routinely used antifungal agents. Aim: This study was undertaken to characterize clinical isolates of Trichosporonsp, using phenotypic methods. Material and Methods: Around 52 isolates of Trichosporon sp. obtained from various samples of patients in Sri Ramachandra Medical College\& Research Institute, Chennai, were considered for the study. The isolates were studied for macroscopic morphology of the colonies on Sabouraud Dextrose Agar (SDA), microscopic morphology by Dalmau technique \& Gram stain, ability to hydrolyze urea, sugar assimilation profile and growth at $37^{\circ} \mathrm{C} \& 0.1 \%$ cycloheximide. Results and Conclusion: Two types of colony morphologies were observed, 47 isolates appeared flat with farinose covering and 5 were cerebriform with radial fissures. All isolates hydrolysed urea, and none of the isolates fermented but only assimilated sugars. The Trichosporon isolates were identified up to species level by phenotypic methods. Still the reliability of identification has to be confirmed with genotypic characterization techniques.
\end{abstract}

Key words: Trichosporon, Colony morphology, Biochemical characteristics

\section{Introduction}

Trichosporonspp. are distributed primarily in tropical and temperate areas. They are present in substrates like soil, water bodies, air, decomposing wood,cattle, scarab beetles, bats, pigeons and even in food particles like cheese. In humans, they constitute a part of normal human flora of the oral cavity, respiratory tract, gastrointestinal tract, skin, and the vagina $[1,2,3]$. They can cause superficial, mucosa-associated or deep-seated infections, collectively called as "Trichosporonosis"[4]. In immuno-competent healthy individuals, Trichosporon spp. may produce superficial infections like white piedra or infections of the skin. However, they can cause invasive infectionsin immunocompromised patients like malignancy patients and transplant recipients [1,2,3]. Invasive trichosporonosis has a high mortality rate and is associated with poor prognosis. The genus Trichosporon belongs to the phylum Basidiomycota, class Hymenomycetes and

Manuscript received: $05^{\text {th }}$ March 2018

Reviewed: $10^{\text {th }}$ March 2018

Author Corrected: $16^{\text {th }}$ March 2018

Accepted for Publication: $22^{\text {nd }}$ March 2018 order Trichosporonales [5]. Cultures on SDA grow as yeast like colonies ranging from white to cream colour, with characteristic farinose or cerebriform surface with radial fissures [6]. On prolonged incubation the colonies may become dry and membranous [7]. Their essential characteristic feature is the presence of budding yeast cells andhyphae, which disarticulates to form arthroconidia[8]. All Trichosporonspp. have cell walls which are multilamellar and septa with rudimentary dolipores [9]. Few other members of this genus possess other morphological structures like appresoria, meristematic cells, etc. that can also be used to differentiate them microscopically. Though the members of the genus Trichosporon are nonfermentative, they are capable of utilizing various carbohydrates and degrade urea by producing the enzyme urease [6]. Microbiological identification of trichosporonosis typically depends on the culture findings and identification of structures matching with Trichosporon spp. like hyphae, pseudohyphae, blastoconidia and arthroconidia in potassium hydroxide $(\mathrm{KOH})$ mount and/or tissue biopsy specimens 


\section{Original Research Article}

[6].Correct identification of this organism till genus and species level is important for epidemiologic reasons and knowledge on resistance characteristics. Invasive trichosporonosis is a life-threatening condition and appropriate antifungal therapy in an early stage of infection is essential for good prognosis $[1,2,3]$.

This study on phenotypic characterization of Trichosporon species is the first of its kind from South India. The data on the morphological and biochemical characterization of 52 Trichosporon sp. isolates, obtained over a five year timeframe has been characterized and analyzed.

\section{Methods}

Strains and Clinical source- We analyzed 52 Trichosporon isolates obtained from different patients of Sri Ramachandra Medical College and Research Institute, Chennai, Tamilnadu, between Jan 2011 \&June 2016. Yeast-like colonies were observed for microscopic characteristics by Gram staining. The isolates which showed blastoconidia, hyphae, pseudohyphae and arthroconidia were provisionally identified as Trichosporonspecies [6] and preserved at $20^{\circ} \mathrm{C}$ on skimmed milk medium until use [10].

Reference strains- The reference strains of Trichosporon species used in our study were procured from Microbial Type Culture Collection and Gene Bank (MTCC), India. The strains used were T. asahii MTCC 6179, T. asteroides MTCC 7632, T. cutaneum var. cutaneum MTCC 1963. The reference strains of Candida species used were Candida albicans ATCC 90028, Candida kruseiATCC 6258.

\section{Morphological and biochemical profile}

Macro morphology- For macroscopy, a small inoculum of an individual, isolated colony of each clinical isolate was inoculated in the centre of SDA plate, and incubated at $25^{\circ} \mathrm{C}$ for 10 days. The study of colony characteristics comprised of the observation of texture, topography and colour.

Micro morphology- Microscopy was studied using Gram stain \&Dalmau technique on cornmeal agar with $1 \%$ tween $80[11]$. The plates were incubated in a moist chamber at $25^{\circ} \mathrm{C}$ for 3-5 days and observed under high power magnification for morphological features like hyphae, pseudohyphae, budding yeast cells, arthroconidia, and specialized structures like appresoria and sarcinae.

Biochemical profile- Biochemical characterization was done as described by De Hoogs et al [6]. The isolates were initially tested for fermentation and assimilation of basic sugars like glucose, maltose, sucrose, lactose, galactose and trehalose. Later they were they were identified till species level, by physiological tests based on assimilation of sugars like Rhamnose, Melibiose, Raffinose, Ribose, Xylose, L-Arabinose, Galactose, Inositol, and growth at $37^{\circ} \mathrm{C} \& 0.1 \%$ cycloheximide [6].

\section{Results}

Sample source- Majority of our isolates were from urine i.e.42 (80.7\%). Around 3(5.7\%) isolates were from blood. For one patient, Trichosporon sp. was isolated from two set of blood cultures and one urine culture. Out of the $2(3.8 \%)$ respiratory isolates, one was bronchoalveolar lavage and one was tracheal aspirate. Around 4 (7.6\%) of the Trichosporonsp. were isolated from pus, and one from peritoneal dialysis fluid (Fig 1).



Fig-1: Sample Source 


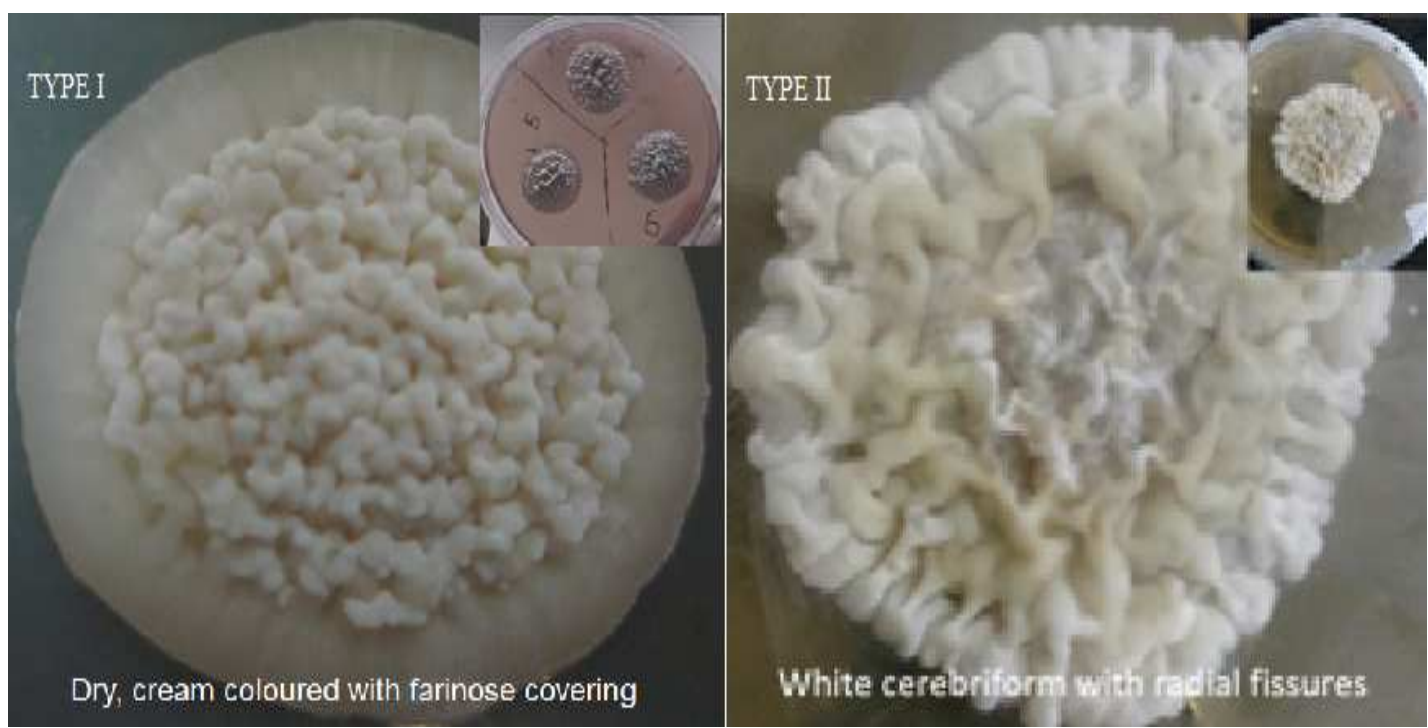

Fig 2: Colony morphology on Sabouraud Dextrose Agar 2A- Type I colony morphology2B- Type II colony morphology

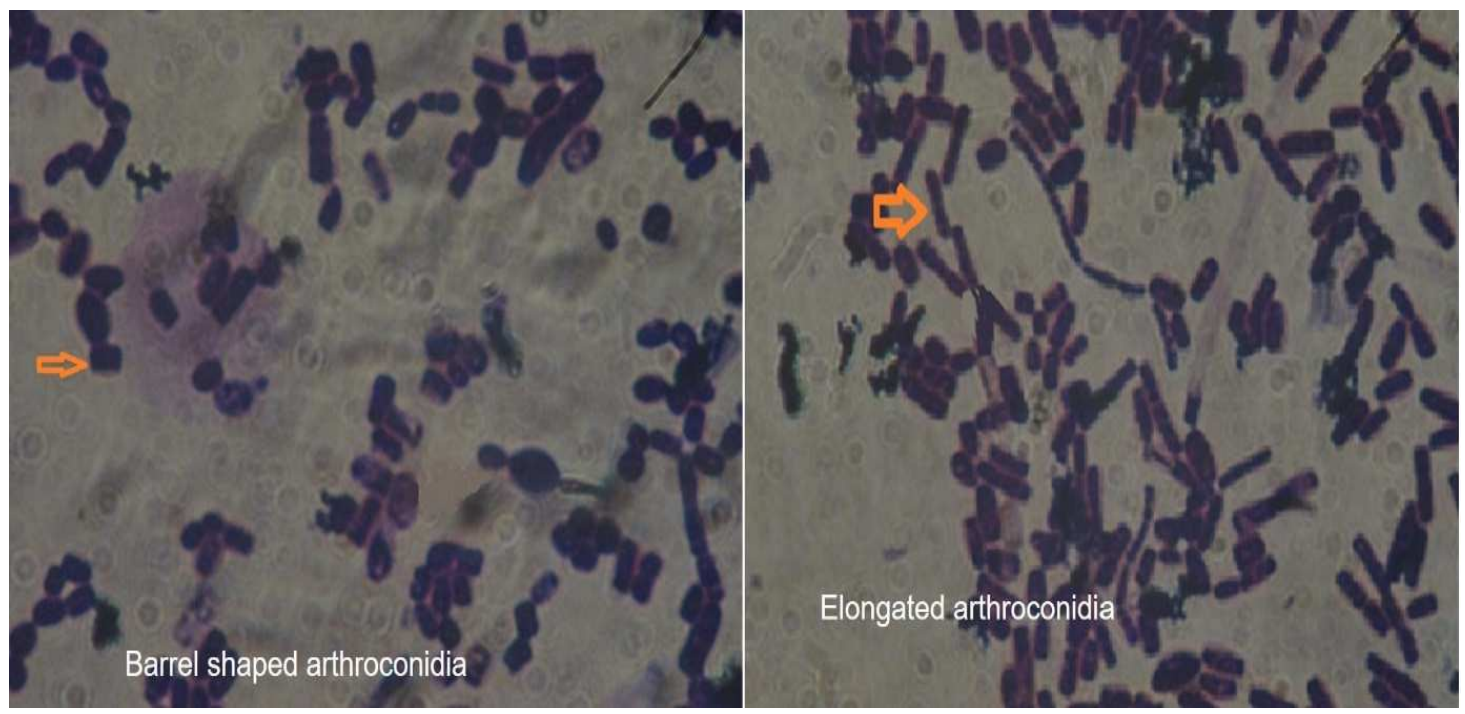

Fig 3A - Gram stain showing Barrel shaped arthroconidia;

3B- Elongated arthroconidia (100x)

\section{Phenotypic Characterization}

Macroscopic appearance- The study of colony morphology of the 52 clinical isolates on SDA at $25^{\circ} \mathrm{C}$ after 10 days of incubation revealed two types of colony morphologies.

Type I - White to Cream, farinose with irregular folds - 42clinical isolates $(80.8 \%)$ (Fig 2 A)

Type II - White, cerebriform with radial furrows-10 clinical isolates (19.2\%)(Fig 2 B)

Type I colonies attained a diameter of around 16-30 mm, whereas type II colonies were smaller with diameter of around 17- $20 \mathrm{~mm}$. Majority of the isolates produced type I colony morphology (80.8\%). Few isolates with cerebriform colony morphology also produced cracking of the medium.

Microscopic appearance- The microscopic observation of the isolates on Cornmeal agar showed two types of observations in gram stain and Dalmau technique.

A- Blastoconidia ,Pseudohyphae and barrel shaped arthroconidia - 44isolates $(84.6 \%)$ (Fig 3A)

B- Blastoconidia, Pesudohyphae and elongated/ cylindrical arthroconidia - 8 clinical isolates (15.4\%) (Fig 3 B) 
All the Type I colonies produced barrel shaped arthroconidia. We did not observe specialized structures like appresoria or meristematic cells for any of our isolates.

Table-1: Biochemical profile and growth characteristics of Trichosporon sp.

\begin{tabular}{|c|c|c|c|c|c|c|c|c|c|c|c|}
\hline \multirow{2}{*}{$\begin{array}{l}\text { No. Of } \\
\text { Isolates } \\
(n=52)\end{array}$} & \multicolumn{8}{|c|}{ Assimilation of } & \multicolumn{2}{|l|}{ Growth } & \multirow{2}{*}{$\begin{array}{l}\text { Final } \\
\text { Identification }\end{array}$} \\
\hline & Rha & Mel & Raff & Rib & Xyl & $\begin{array}{l}\text { L- } \\
\text { Arab }\end{array}$ & Galac & Ino & $\begin{array}{l}0.1 \% \\
\text { Cyclohex } \\
\text { i }\end{array}$ & $37^{\circ} \mathrm{C}$ & \\
\hline 28 & + & - & - & - & - & + & - & - & + & + & T. asahii \\
\hline 9 & + & - & - & + & - & + & - & - & + & + & T. asahii \\
\hline 7 & + & - & - & - & + & + & - & - & + & + & T. asahii \\
\hline 3 & - & - & - & - & - & + & - & + & + & + & T. asteroides \\
\hline 2 & + & + & + & + & + & + & - & + & - & - & T. cutaneum \\
\hline
\end{tabular}

Note: Three isolates produced inconclusive results hence not shown in table.

Rha- Rhamnose; Mel- Melibiose; Raff- Raffinose; Rib- Ribose; Xyl- Xylose; L-Arab- L- Arabinose; Galac- Galactose; Ino- Inositol; $0.1 \%$ cyclohexi- $0.1 \%$ cycloheximide.

Biochemical Identification- The isolates were identified phenotypically according to the biochemical characteristics as per De Hoogs et al [6]. Fermentation was absent in all the isolates for the basic sugars testedlike glucose, maltose, sucrose, lactose, galactose and trehalose. The sugar assimilation profile and growth characteristics for speciation of the isolates is listed in Table 1. For about 28 isolates, growth was positive in rhamnose, L-arabinose, $0.1 \%$ cycloheximide and $37^{\circ} \mathrm{C}$, identifying them as T.asahii. Growth was present in ribose for 9 isolates; and in xylose for 7 isolates, apart from the above-mentioned sugars. Since the growth in xylose and ribose may be variable for T.asahii, all the 44 isolates were identified as T.asahii. Three isolates grew in L-arabinose, inositol, $0.1 \%$ cycloheximide and $37^{\circ} \mathrm{C}$, but did not grow in rhamnose. They were identified as T.asteroides. Two isolates grew in all the sugars except galactose, $0.1 \%$ cycloheximide \& $37^{\circ} \mathrm{C}$, which were identified as T.cutaneum. For aroundthree isolates, the reactions were inconclusive.

\section{Discussion}

Trichosporonsp has been so far documented as the second most common cause of invasive yeast infections in humans, next to the genus Candida[12]. They are newly evolving opportunistic pathogens causing invasive infections in immuno-compromised patients $[13,14]$.Theyremain associated with high mortality rates, since they do not respond to treatment with routinely used antifungal agents. Such infections pose a greatchallenge fordiagnosis $[4,13]$.

Yeast identification is usually done based on evaluation of macroscopic colony morphology, microscopic appearance, physiological and biochemical characteristics. The physiological and biochemical tests which areroutinely used foridentification are, fermentation of and growth on carbon sources, growth on nitrogen sources, growth at various temperatures and hydrolysis of urea[14,15].

In this study, we have analysed the phenotypic characters of 52 Trichosporon clinical isolates. Phenotypic characterization was done by observing colony morphology, microscopic morphology,and biochemical\& growth characteristics. Two types of colonies morphologies were exhibited by 52 clinical isolates (cream to white farinose and white cerebriform i.e type I \& II). Isolates which were phenotypically identified as $\mathrm{T}$ asahii mainly exhibited type I colony morphology. Another author Ichikawa et al had observed 4 types of colonies produced by $\mathrm{T}$ asahii, whereas Ahmad et al observed only single type of colony morphology byT.asahii $[16,17]$.This difference could be due to the variations in the sample sources used in these studies [18]. In another study,Ichikawa et al.,2003, demonstrated that phenotypic switching of colony morphology among T.asahii isolates directly contributed to their virulence by facilitating invasion and escape from host defenses [16].

All the 44T.asahii isolates produced barrel shaped arthroconidia. This finding was similar to the microscopic morphology as described by De Hoog et al [6].

The biochemical profile and growth characteristics identified our isolates as T.asahii(44/ 52), 


\section{Original Research Article}

T.asteroides(3/52), T.cutaneum(2/52)and remaining three isolates produced inconclusive results, and hence they could not be identified. The collective use of light microscopic evaluationand biochemical characteristicshelps in the identification of majority of the species causing human infections. Nevertheless, new species of yeasts causing opportunistic infections has increased during the past few years, making the identification by the routinely used conventional methods difficult, even for experts in the field of mycology. Furthermore, these conventional identification methods can be laborious producing inconclusive results, leading to misidentifications particularly for uncommon pathogenic species. Comparison of biochemical and physiological characteristics of Trichosporon spp. by different laboratories had also revealed significant discrepancies in the results generated by other researchers [19,20]. Hence, the phenotypic characterization results should always be confirmed with genotypic methods for identification of Trichosporonsp.

Though performing a slide micro-culture to demonstrate the presence of arthroconidia and urease test are very valuablemethods for the screening of Trichosporon spp., other morphological features and biochemical tests do not allow the precise species level identification of Trichosporon isolates.

\section{Conclusion}

T.asahii is the most common clinical isolate identified phenotypically in our study. We did not isolate wide spectrum of medically important species of the genus Trichosporon. This may which may be attributed to the nature of the clinical specimen culture din our study. We did not have samples from superficial sites like skin and hair, which may have varied species range. Due to inconsistency, difficulty in identification and failure to identify new emerging species, we can conclude that conventional methods for yeast identification should always be followed by molecular methods for confirmation of the findings.

\section{Funding: Nil, Conflict of interest: None \\ Permission of IRB: Yes}

\section{References}

1. Chagas-Neto TC, Chaves GM, Melo ASA, et al. Bloodstream infections due to Trichosporonspp: species distribution, Trichosporonasahii genotypes determined on the basis of ribosomal DNA intergenic spacer 1 sequencing, and antifungal susceptibility testing. J ClinMicrobiol, 2009; 47(4): 1074-81.
2. Ruan SY, Chien JY, Hsueh PR.Invasive trichosporonosis caused by Trichosporonasahii and other unusual Trichosporon species at a medical center in Taiwan. Clin Infect Dis. 2009 Jul 1;49(1):e11-7. doi: 10.1086/599614.

3. Suzuki K, Nakase K, Kyo T, Kohara T, Sugawara Y, Shibazaki T, Oka K, Tsukada T, Katayama N.FatalTrichosporonfungemia in patients with hematologic malignancies. Eur J Haematol. 2010 May;84(5):441-7. doi: 10.1111/j.16000609.2010.01410.x.Epub 2010 Jan 13.

4. Colombo AL, Padovan AC, Chaves GM. Current knowledge of Trichosporon spp. and Trichosporonosis. ClinMicrobiol Rev. 2011 Oct;24(4):682-700. doi: 10.1128/CMR.00003-11.

5. Fell JW, Boekhout T, Fonseca A, Scorzetti G, Statzell-Tallman A.Biodiversity and systematics of basidiomycetousyeasts as determined by largesubunitrDNAD1/D2domainsequence analysis. Int $\mathrm{J}$ SystEvolMicrobiol. 2000 May;50 Pt 3:1351-71.

6. De Hoog G. S, J Guarro, J Gene, M. J. Figueres. Atlas of clinical fungi. Centraalbureauvoor Schimmelcultures Utrecht, The Netherlands, and UniversitatRoviraiVirgili, Reus, Italy.2000.

7. Kwon-Chung K. J, J. E. Bennett. Medical mycology. Lea \&Febiger, Philadelphia, PA.1992.

8. Lacaz CS, Porto E, Martins JEC. MicologiaMédica. Fungos, actinomicetos e algas de interessemédico. 1984; 7th ed; Sarvier, São Paulo, 479 pp.

9. Guého E, de Hoog GS, Smith MT. Neotypification of the genus Trichosporon. Antonie Van Leeuwenhoek. 1992 May;61(4):285-8.

10. Magalhães AR, Mondino SS, Silva Md, Nishikawa MM.Morphological and biochemicalcharacterization of the aetiologicalagents of white piedra. Mem Inst Oswaldo Cruz. 2008 Dec;103(8):786-90.

11. McGinnis M. R. Mycology. In Clinical Microbiology Procedures Handbook.Edited by H. D. Isenberg. Washington, DC: American Society for Microbiology.1994; Section 6: pp. 6.1.1-6.1.6.1.12.

12. Fleming RV, Walsh TJ, Anaissie EJ. Emerging and less common fungal pathogens. Infect Dis Clin North Am. 2002 Dec;16(4):915-33, vi-vii. 


\section{Original Research Article}

13. Walsh TJ, Groll A, Hiemenz J, Fleming R, Roilides E, Anaissie E. Infections due to emerging and uncommon medically important fungal pathogens. ClinMicrobiol Infect. 2004 Mar;10 Suppl 1:48-66.

14. Barnett J. A, R. W. Payne, D. Yarrow. Yeasts: characteristics and Identification. Cambridge University Press, Cambridge, United Kingdom. 2000; 3rd ed.

15. Kurtzman C. P, J. W. Fell. The yeasts. A taxonomic study. Elsevier, Amsterdam, The Netherlands.1998.

16. Ichikawa T, Sugita T, Wang L, Yokoyama K, Nishimura K, Nishikawa A. Phenotypic switching and beta-N-acetylhexosaminidase activity of the pathogenic yeast Trichosporonasahii. Microbiol Immunol. 2004;48(4):237-42.
17. Ahmad S, Al-Mahmeed M, Khan ZU. Characterization of Trichosporon species isolated from clinical specimens in Kuwait. J Med Microbiol. 2005 Jul;54(Pt 7):639-46.

18. Lee J. W, Melcher G. A, Rinaldi M. G, Pizzo P. A, Walsh T. J. Patterns of morphologic variation among isolates of Trichosporonbeigelii. J ClinMicrobiol. 1990; 28: 2823-2827.

19. Pincus DH, Orenga S, Chatellier S. Yeast identification--past, present, and future methods. Med Mycol. 2007 Mar;45(2):97-121.

20. Sugita T, Nishikawa A, Shinoda T. Rapid detection of species of the opportunistic yeast Trichosporon by PCR. J ClinMicrobiol. 1998 May;36(5):1458-60.

\section{How to cite this article?}

Premamalini T, Anitha S., Mohana Priya, Kindo A. J. Phenotypic characterization of Trichosporon species from clinical isolates. Int J Med Res Rev 2018;6 (03):153-158. doi:10.17511/ijmrr. 2018.i03.04. 